\title{
S-Shaped Complementary Split Ring Resonators and Their Application to Compact Differential Bandpass Filters with Common-Mode Suppression
}

\author{
Ali K. Horestani, Student Member, IEEE, Miguel Durán-Sindreu, Member, IEEE, Jordi Naqui, Student \\ Member, IEEE, Christophe Fumeaux, Senior Member, IEEE, and Ferran Martín, Fellow, IEEE
}

\begin{abstract}
This letter presents an S-shaped complementary split ring resonator (S-CSRR) for application in compact differential filters. The working principle of the proposed S-CSRR is explained and a circuit model is developed and validated through electromagnetic simulations. It is shown that an S-CSRR-loaded differential microstrip line with series gaps can be used in the design of compact differential bandpass filters with commonmode suppression. The filter design procedure is explained and the theoretical concept is validated through fabrication and measurement of a compact $\left(0.09 \lambda_{g} \times 0.25 \lambda_{g}\right)$ third-order differential bandpass filter with common-mode suppression.
\end{abstract}

\section{INTRODUCTION}

Transmission of high-speed digital and analog signals in differential mode is proven to be the most efficient method to increase the signal immunity to environmental noise, electromagnetic interference (EMI) and crosstalk. However, in practical differential systems, the presence of some level of common-mode noise is unavoidable [1]. Thus, a differentialto-differential balanced filter that passes the desired frequency response of the differential signal, while suppressing the common-mode noise is one of the main building blocks in balanced circuits. A first category of realizations includes filters that are composed of a differential bandpass filter in combination with a dedicated structure for common-mode suppression [1]-[3]. A second category includes structures which combine differential filtering and common-mode suppression, and are thus usually more compact [4]-[7]. This paper proposes the dual counterpart of S-shaped split ring resonator [8], i.e., S-shaped complementary split ring resonator (S-CSRR) for application in the design of differential filters. It is shown that compared to the conventional configuration of CSRRs in differential microstrip technology, the proposed resonator benefits from a high level of miniaturization when

This work has been supported by the Australian Research Council under project DP120100661, and by MINECO (Spain) under projects TEC201017512, CSD2008-00066 and TEC2011-13615-E. Thanks are also given to AGAUR-Generalitat de Catalunya for partially funding this research activity through the project 2009SGR-421. Jordi Naqui is also in debt to MINECO (Spain) for supporting his work through the FPU grant AP2010-0431.

Ali K. Horestani is with the School of Electrical \& Electronic Engineering, The University of Adelaide, Adelaide, SA 5005, Australia and also with the Ministry of Science, Research and Technology, Tehran, Iran. He was a visiting researcher at GEMMA/CIMITEC when this work was performed. (e-mail: akarami@eleceng.adelaide.edu.au).

C. Fumeaux is with the School of Electrical \& Electronic Engineering, The University of Adelaide, Adelaide, SA 5005, Australia.

Miguel Durán-Sindreu, Jordi Naqui, and Ferran Martín are with GEMMA/CIMITEC, Departament d'Enginyeria Electronica, Universitat Autonoma de Barcelona, 08193 Bellaterra, Spain (e-mail: Ferran.Martin@uab.es).

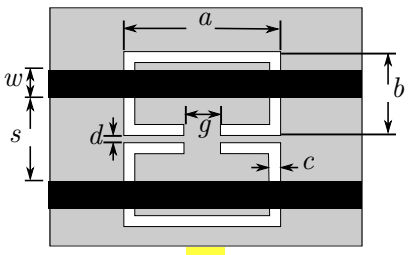

(a)

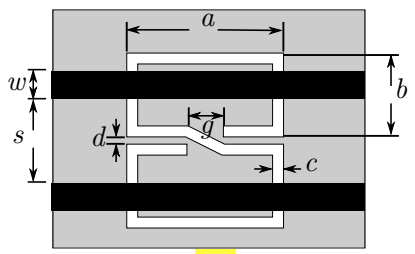

(b)
Fig. 1. A differential microstrip line loaded with (a) a pair of rectangular single ring CSRRs, and (b) an S-shaped CSRR.

excited by the contra-directional electric fields of a differential microstrip line. More importantly, it is shown that S-CSRRs can be used in the design of bandpass filters that suppress the common-mode noise inherently.

\section{Principle of Miniaturization}

Fig. 1(a) shows the typical configuration of a CSRR-loaded differential microstrip line, with a pair of CSRRs etched in the ground plane under each strip of the differential line. In this configuration, regardless of the signal mode, the CSRRs are exited by the time-varying axial electric fields of the microstrip line. Thus, the propagation of electromagnetic waves is inhibited in a narrow band around the CSRR resonance. It was shown in [3] that an array of single CSRRs aligned with the symmetry plane of the differential line can be used for common-mode suppression, while the differential signal is unaffected. The key to this application is that while the CSRRs are exited by the axial electric fields in common-mode, the electric field components of the differential signals, which are contra-directional, cancel each other and the CSRRs are not excited by this mode. In contrast, the fundamental resonance of the proposed S-shaped CSRR, shown in Fig. 1(b), is activated if the connected rings of the S-CSRR are excited by contra-directional axial electric fields of the loaded differential microstrip line. Thus, an S-CSRR can be used to efficiently inhibit the propagation of differential signals in a narrow frequency band near the resonance frequency of the resonator.

Fig. 2 shows the simulated transmission and reflection coefficients of the S-CSRR loaded structure in differential mode. For the electromagnetic simulations, a $0.635 \mathrm{~mm}$ thick Rogers RO3010 substrate with relative permittivity of $\epsilon_{r}=10.2$ is used. The width of the differential microstrip lines is $w=0.5 \mathrm{~mm}$ and the space between the strips is $s=5.75 \mathrm{~mm}$, which correspond to a $50 \Omega$ odd-mode characteristic impedance. The S-CSRR's dimensions are as follows: $a=6.2 \mathrm{~mm}, b=5.7 \mathrm{~mm}, c=0.2 \mathrm{~mm}, d=0.2 \mathrm{~mm}$, and $g=1 \mathrm{~mm}$. For comparison, the simulated odd-mode 


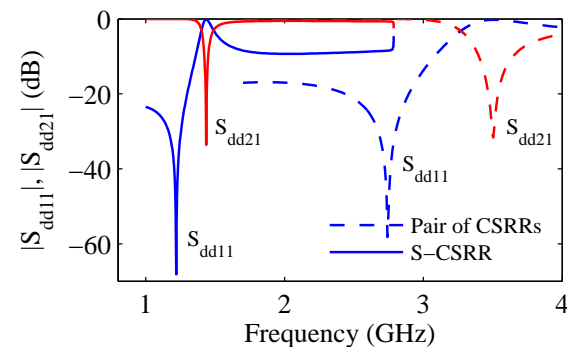

Fig. 2. Comparison between the simulated transmission and reflection coefficients (differential mode) of the differential microstrip line loaded with an S-CSRR ( solid lines) and a pair of CSRRs with the same physical size (dashed lines).

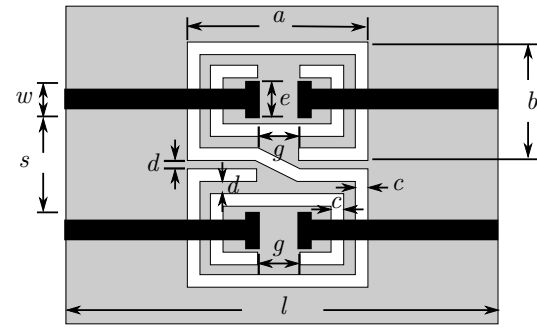

(a)

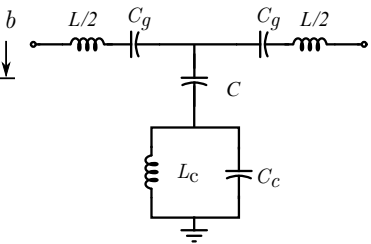

(b)
Fig. 3. (a) Layout of an S-CSRR/gap loaded differential line. The dimensions of the structure are as follows: $a=9.8 \mathrm{~mm}, b=7 \mathrm{~mm}, c=0.8 \mathrm{~mm}$, $d=0.2 \mathrm{~mm}, g=0.6 \mathrm{~mm}, w=0.5 \mathrm{~mm}, s=5.75 \mathrm{~mm}, e=2 \mathrm{~mm}$, and $l=22 \mathrm{~mm}$. (b) Equivalent lumped element circuit model of the unit cell for both differential and common modes.

frequency response of the differential microstrip line loaded with a pair of CSRRs with the same physical dimensions as the rings of S-CSRR is also depicted in the figure. The simulation results show that the resonance frequency of the structure with the pair of CSRRs is $f=3.5 \mathrm{GHz}$, while the S-CSRR resonates at a much lower frequency $(1.43 \mathrm{GHz})$. The comparison shows that a high level of miniaturization is achieved by modifying the resonator shape to an S-shaped CSRR.

\section{Modeling OF S-CSRR/GAP-LOAdED LINE}

The bandstop behavior of an S-CSRR-loaded differential microstrip line can be interpreted as due to the negative permittivity of the structure in the vicinity of the S-CSRR's fundamental resonance. This bandstop behavior can be switched to a bandpass behavior simply by introducing series capacitive gaps in the strips of the differential line, as shown in Fig. 3(a) [9]. Note that in this structure, inner split slot rings are added to the S-CSRR to achieve more compactness.

In order to study the behavior of the S-CSRR/gap-loaded differential microstrip line, we now focus on the circuit model and parameter extraction of the structure, in both differential and common modes. Fig. 3(b) depicts the proposed lumped element circuit model for the structure of Fig. 3(a), where $L$ and $C$ are the per-section equivalent inductance and capacitance of the differential line with the presence of the SCSRR, and $C_{g}$ models the line's capacitive gaps. The S-CSRR is modeled with an equivalent capacitance $C_{c}$ and an equivalent inductance $L_{c}$. This model is valid for both differential and common modes, however with different element values. Indeed, while the fundamental resonance of the S-CSRR is excited by differential mode (Fig. 4(a)), the common mode excites the S-CSRR's higher order resonance (Fig. 5(a)).

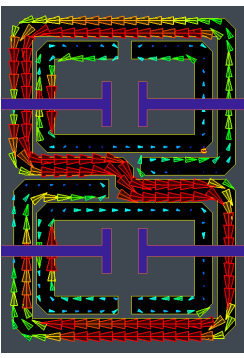

(a)
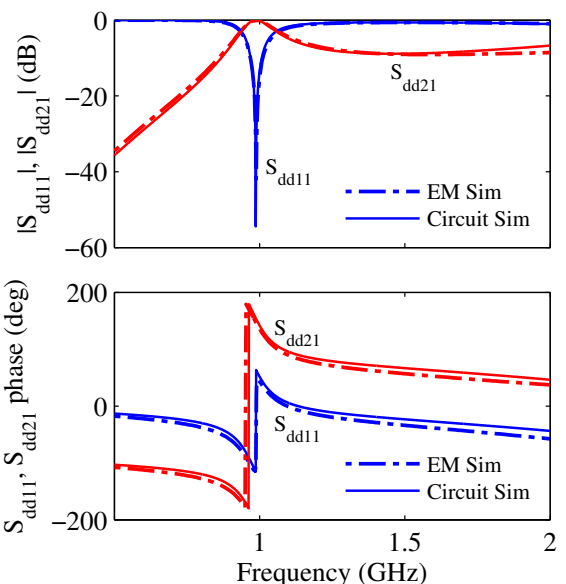

(b)
Fig. 4. (a) Magnetic current distribution in differential mode, and (b) comparison between the amplitude (top) and phase (bottom) of the differential-mode transmission and reflection coefficients from the electromagnetic simulation of the structure of Fig. 3 and those from the proposed circuit model.

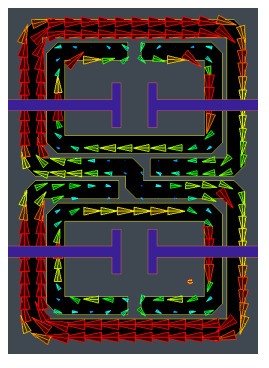

(a)
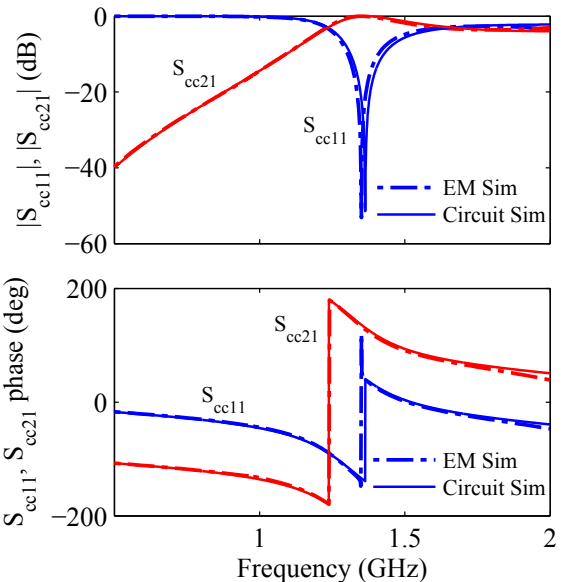

(b)
Fig. 5. (a) Magnetic current distribution in common mode, and (b) comparison between the amplitude (top) and phase (bottom) of the common-mode transmission and reflection coefficients from the electromagnetic simulation of the structure of Fig. 3 and those from the proposed circuit model.

Using the procedure explained in [10] the parameters of the circuit model for differential and common modes are extracted from the electromagnetic simulations of the structure of Fig. 3(a). Dimensions of the structure are indicated in the caption of the figure, and the same substrate as in the Section II is used for electromagnetic simulations. The extracted parameters for differential mode are: $L=8 \mathrm{nH}$, $C=70 \mathrm{pF}, C_{g}=0.33 \mathrm{pF}, C_{c}=1.2 \mathrm{pF}$, and $L_{c}=10 \mathrm{nH}$, whereas the parameters for the common mode are: $L=4 \mathrm{nH}$, $C=105 \mathrm{pF}, C_{g}=0.46 \mathrm{pF}, C_{c}=1.5 \mathrm{pF}$, and $L_{c}=4.2 \mathrm{nH}$. Figs. 4(b) and 5(b) demonstrate a good agreement between the transmission and reflection coefficients of the circuit model and electromagnetic simulations for both modes, validating the proposed circuit model. Note that the structure's passband for differential mode is centered at $f=1 \mathrm{GHz}$, whereas the passband for the common mode is located at $f=1.4 \mathrm{GHz}$. This feature can be used in the design of differential bandpass filters with common-mode suppression. 


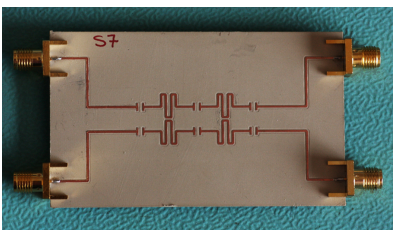

(a)

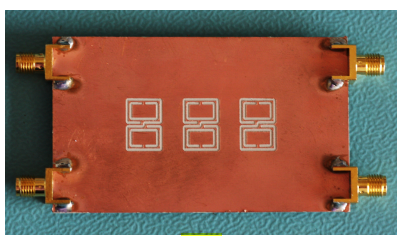

(b)
Fig. 6. (a) Top and (b) bottom photograph of the fabricated third order differential bandpass filter with common-mode suppression.

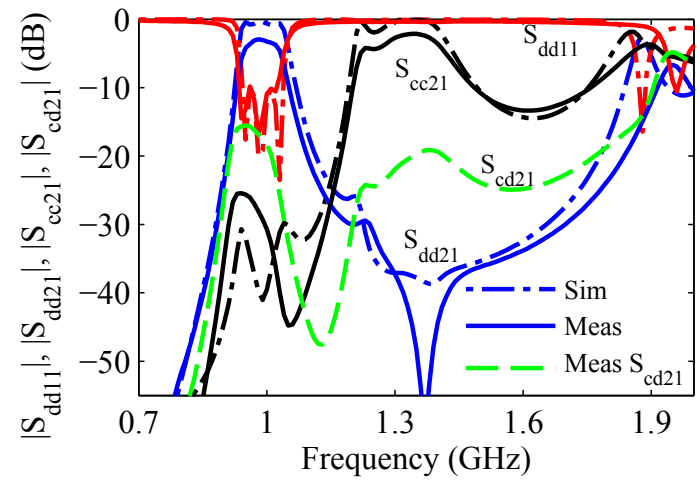

Fig. 7. Comparison between simulated (lossless) and measured transmission and reflection coefficients of the third-order differential filter with commonmode suppression.

\section{BANDPASS FILTER WiTh COMMON-MODE SUPPRESSION}

To illustrate the potential application of the S-CSRR in the design of a differential bandpass filter with common-mode suppression, a third-order periodic (although, periodicity is not mandatory from the design point of view) bandpass filter with fractional bandwidth of $10 \%$ and $0.16 \mathrm{~dB}$ in-band ripple is designed and synthesized for operation roughly centered around the frequency of $1 \mathrm{GHz}$. The focus of the design procedure is simply placed on the differential characteristics of the filter, while the common-mode is intrinsically suppressed by the proposed structure.

The structure of Fig. 3, which can be considered as a distributed resonator with two $\lambda / 8$ feed lines, is used as the filter's unit cell. The feed lines of each unit cell in conjunction with those of the adjacent cells act as $90^{\circ}$ impedance inverters with normalized impedance of $K=1$. Based on the theory of impedance inverters, the fractional bandwidth of the filter can be controlled by the reactance slope of the unit cell, excluding the feed lines [11], which is in turn controlled by gap dimensions [9]. Thus, a parametric analysis has been applied in the design process in order to determine the optimum gap dimensions. The next step is to optimize the length of the feed lines to achieve a $90^{\circ}$ phase shift from port one to port two of the unit cell at resonance frequency. Finally, the filter is realized by cascading the optimized unit cells.

The photograph of the fabricated filter, using the same substrate as in Sec. II and the dimensions in the caption of Fig. 3, is depicted in Fig. 6. The impedance inverters are meandered to achieve more compactness. The filter is as small as $0.09 \lambda_{g} \times 0.25 \lambda_{g}$, where $\lambda_{g}$ is the guided wavelength at $f=$ $1 \mathrm{GHz}$. A comparison between electromagnetic simulation and measured frequency response of the filter in differential and common modes is depicted in Fig. 7. The figure shows that the structure acts as a differential bandpass filter with
TABLE I

A COMPARISON OF VARIOUS DIFFERENTIAL BANDPASS FILTERS.

\begin{tabular}{l|c|l|l|l|l}
\hline Ref. & $n$ & $\begin{array}{l}\text { CMRR in } \\
\text { passband }\end{array}$ & $\frac{f_{1 c c}}{f_{1 d d}}$ & $\frac{f_{2 c c}}{f_{2 d d}}$ & Electrical Size \\
\hline$[4]$ & 3 & $>24 \mathrm{~dB}$ & 0.96 & 1.09 & $0.5 \lambda_{g} \times 0.5 \lambda_{g}$ \\
{$[5]$} & 3 & $>18 \mathrm{~dB}$ & 0.93 & 1.08 & $0.5 \lambda_{g} \times 0.5 \lambda_{g}$ \\
{$[7]$} & 3 & $>25 \mathrm{~dB}$ & 0 & 1.04 & $0.15 \lambda_{g} \times 0.3 \lambda_{g}$ \\
This work & 3 & $>25 \mathrm{~dB}$ & 0 & 1.15 & $0.09 \lambda_{g} \times 0.25 \lambda_{g}$ \\
\hline
\end{tabular}

$2.8 \mathrm{~dB}$ insertion loss at central frequency, common-mode suppression better than $-25 \mathrm{~dB}$, and common-to-differential mode conversion better than $-16 \mathrm{~dB}$. A comparison with other differential filters in terms of performance and size is provided in Table I, where $f_{1 d d}$ and $f_{2 d d}$ are lower and upper $-3 \mathrm{~dB}$ cutoff frequencies in differential mode, and $f_{1 c c}$ and $f_{2 c c}$ are lower and upper $-20 \mathrm{~dB}$ cutoff frequencies in common-mode stopband.

\section{Conclusion}

This work has presented an S-shaped complementary split ring resonator for application in differential microstrip technology. Size reduction as compared to CSRRs has been achieved. More importantly, it has been shown that an S-CSRR-loaded microstrip line with series capacitive gaps can be used as a unit cell for differential bandpass filters with common-mode suppression. In-band common-mode rejection ratio better than $-25 \mathrm{~dB}$ has been achieved.

\section{REFERENCES}

[1] S. Wu, C.-H. Tsai, T. Wu, and T. Itoh, "A novel wideband commonmode suppression filter for gigahertz differential signals using coupled patterned ground structure," IEEE Trans. Microw. Theory Tech., vol. 57, no. 4, pp. 848-855, Apr. 2009.

[2] C.-H. Tsai and T. Wu, "A broadband and miniaturized common-mode filter for gigahertz differential signals based on negative-permittivity metamaterials," IEEE Trans. Microw. Theory Tech., vol. 58, no. 1, pp. 195-202, Jan. 2010

[3] J. Naqui, A. Fernandez-Prieto, M. Durán-Sindreu, F. Mesa, J. Martel, F. Medina, and F. Martín, "Common-mode suppression in microstrip differential lines by means of complementary split ring resonators: theory and applications," IEEE Trans. Microw. Theory Tech., vol. 60, no. 10, pp. 3023-3034, Oct. 2012.

[4] T. B. Lim and L. Zhu, "A differential-mode wideband bandpass filter on microstrip line for UWB application," IEEE Trans. Microw. Theory Tech., vol. 19, no. 10, pp. 632-634, Oct. 2009.

[5] — , "Highly selective differential-mode wideband bandpass filter for UWB application," IEEE Trans. Microw. Theory Tech., vol. 21, no. 3, pp. 133-135, Mar. 2011.

[6] S. Shi, W.-W. Choi, W. Che, K.-W. Tam, and Q. Xue, "Ultra-wideband differential bandpass filter with narrow notched band and improved common-mode suppression by DGS," IEEE Trans. Microw. Theory Tech., vol. 22, no. 4, pp. 185-187, Apr. 2012.

[7] P. Velez, J. Naqui, A. Fernandez-Prieto, M. Durán-Sindreu, J. Bonache, J. Martel, F. Medina, and F. Martín, "Differential bandpass filter with common-mode suppression based on open split ring resonators and open complementary split ring resonators," IEEE Trans. Microw. Theory Tech., vol. 23, no. 1, pp. 22-24, Jan. 2013.

[8] A. K. Horestani, M. Durán-Sindreu, J. Naqui, C. Fumeaux, and F. Martín, "Coplanar waveguides loaded with S-shaped split ring resonators (S-SRRs) and their application to compact bandpass filter design," IEEE Trans. Microw. Theory Tech., submitted for publication.

[9] M. Gil, J. Bonache, I. Gil, J. García-García, and F. Martín, "On the transmission properties of left-handed microstrip lines implemented by complementary split rings resonators," International Journal of Numerical Modelling: Electronic Networks, Devices and Fields, vol. 19, no. 2, pp. 87-103, Mar. 2006.

[10] J. Bonache, M. Gil, I. Gil, J. García-García, and F. Martín, "On the electrical characteristics of complementary metamaterial resonators," IEEE Trans. Microw. Theory Tech., vol. 16, no. 10, pp. 543-545, Oct. 2006.

[11] J. S. Hong and M. J. Lancaster, Microstrip Filters for RF/Microwave Applications. New York: Wiley, 2001. 